\title{
MMP12 is a potential therapeutic target for Adamantinomatous craniopharyngioma: Conclusions from bioinformatics analysis and in vitro experiments
}

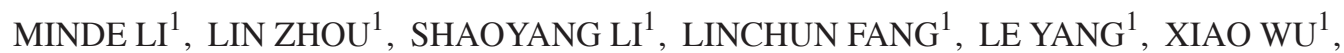 \\ CHENXING YANG $^{1}$, YOUYUAN BAO ${ }^{1}$, SIHAI LAN ${ }^{1}$, ZHIGAO TONG ${ }^{1}$, SUYUE ZHENG ${ }^{1}$, \\ BIN TANG $^{1}$, ERMING ZENG ${ }^{1}$, SHENHAO XIE ${ }^{1},{\text { CHENG } \text { CHEN }^{2} \text { and TAO HONG }}^{1}$ \\ ${ }^{1}$ Department of Neurosurgery, The First Affiliated Hospital of Nanchang University, Nanchang, Jiangxi 330006; \\ ${ }^{2}$ Department of Rehabilitation Medicine, Lushan Sanatorium, Jiujiang, Jiangxi 332000, P.R. China
}

Received September 22, 2020; Accepted April 20, 2021

DOI: 10.3892/ol.2021.12797

\begin{abstract}
Adamantinomatous craniopharyngioma (ACP) is considered a benign intracranial tumor, but it can also exhibit aggressive characteristics. Due to its unique location in the suprasellar, which brings it close to important nerves and vascular structures, ACP can often lead to significant neuroendocrine diseases. The current treatments primarily include surgical intervention, radiation therapy or a combination of the two, but these can lead to serious complications and adversely affect the quality of life of patients. Thus, it is important to identify effective and safe alternatives. Recently, studies have focused on the tumor genome, transcriptome and proteome in an attempt to identify potential therapeutic targets for clinical use. However, studies on this region of the $\mathrm{CP}$ are limited; thus, the present study focused on this region. The GSE94349 and GSE68015 datasets were downloaded from the Gene Expression Omnibus database and analyzed. In the in vitro studies, the effect of the matrix metalloproteinase (MMP)12 inhibitor, MMP408, on cell proliferation and protein expression was assessed. The results demonstrated that MMP408 effectively inhibited cell proliferation and migration of ACP cells, and decreased the expression levels of the related proteins. Thus, MMP12 may be used as a potential therapeutic target for the treatment of ACP.
\end{abstract}

\section{Introduction}

Adamantinomatous craniopharyngioma (ACP) is a benign tumor, but may exhibit invasive characteristics clinically $(1,2)$.

Correspondence to: Professor Tao Hong, Department of Neurosurgery, The First Affiliated Hospital of Nanchang University, 17 Yongwaizheng Street, Nanchang, Jiangxi 330006, P.R. China

E-mail: ht2000@vip.sina.com

Key words: adamantinomatous craniopharyngioma, matrix metalloproteinase 12 , targeted therapy drug inhibitor, bioinformatics
ACP occurs in saddle areas and is hypothesized to develop from the Rathke's pouch $(1,2)$. For $\mathrm{CP}$, following the first successful removal via surgery $\sim 100$ years ago, coupled with advances in surgical techniques and endocrine care, the mortality rate of patients has decreased from $~ 100-5 \%$ (3-6). Despite advancements in the treatment of ACP, there is still a high risk of recurrence and surgical complications due to its unique anatomical location (close proximity to the optic chiasma, optic nerve, pituitary stalk, internal carotid artery, hypothalamus and middle cerebral artery) and high risk of compression or perforation of arteries and other important structures, including the hypothalamus, pituitary stalk and optic chiasm $(7,8)$. Thus, it is important to identify alternative treatments. Recently, considerable efforts have been made to analyze the genome, transcriptome and proteome of these tumors to identify potential therapeutic targets (9-11). However, studies on this region of the $\mathrm{CP}$ are limited. Understanding the molecular mechanisms underlying the development of ACP, as well identifying potentially relevant biomarkers of metastasis may assist in the management of patients.

Currently, high-throughput data analysis technologies, including RNA sequencing and gene expression data chip technology, are widely used to study the molecular mechanisms underlying tumorigenesis. Abnormal mRNA expression and differentially expressed genes (DEGs) can be detected by mRNA expression chips (9-11). Microarray technology has been used to identify DEGs, some of which have assisted in understanding the development and progression of malignant tumors (12). However, studies using gene expression microarray platforms to determine the gene expression profile in ACP tissues are limited, despite being commonly used for other types of tumors (12-14). The integrated gene expression database, Gene Expression Omnibus (GEO) provides a method for mining multiple tumor gene expression profiles for bioinformatics analysis (15). Matrix metalloproteinase (MMP)12, also termed macrophage metalloelastase, is a type of metalloproteinase secreted as part of an inflammatory response, and was initially identified in 1975 (16-18). Previous studies have demonstrated that MMP12 gene knockout in homozygous mice results in physiological abnormalities in macrophages, 
such as a decrease in sensitivity to cigarette smoke, and mice had smaller litter sizes $(19,20)$. Furthermore, a recent study reported that inhibition of MMP12 may be a novel means of antiviral therapy (21).

MMP12 is expressed in several types of cancer, including colorectal cancer, gastric cancer, lung cancer and liver cancer, as well as ACP (22-26). MMP12 can degrade the extracellular matrix and vascular components, thus promoting tumor invasion and migration (26,27). Conversely, other study have reported that MMP12 may also inhibit tumor growth, invasion and migration $(28,29)$. MMP12 is expressed in macrophages, as well as tumor cells (29). Recent studies have demonstrated that MMP12 expressed in the periphery of the tumor suppresses tumor growth, whereas that expressed within the tumor promotes its growth $(28,29)$. Thus, investigating MMP12 expression in ACP, and whether inhibition of MMP12 in ACP can inhibit the proliferation of tumor cells, is considered important.

MMP408 is an effective selective MMP12 inhibitor $(30,31)$. Previous studies have reported that MMP408 can exert specific inhibitory effects on MMP12 at both mRNA and protein levels, and MMP12 can affect the inflammatory response due to its expression on macrophages $(30,31)$. Inflammation serves an important role in the occurrence and development of ACP (32). Thus, it was speculated that MMP12 may exhibit beneficial effects for management of ACP.

In the present study, an integrated bioinformatics approach was used to identify the DEGs between ACP and normal tissues, based on datasets obtained from the GEO database (GSE94349 and GSE68015), to identify novel biomarkers associated with progression and pathogenesis of ACP. The present study focused on MMP12 based on the findings of a previous study (33). In addition, the effects of inhibiting MMP12 in vitro were assessed.

\section{Materials and methods}

Microarray data. ACP gene expression databases were obtained from NCBI GEO (ncbi.nlm.nih.gov/geo). For the present study, two GEO datasets, GSE94349 (34) and GSE68015 (35), which contained 48 tumor samples and 48 normal samples were used.

DEGs. The limma package (versions 3.46.0; Bioconductor) in $\mathrm{R}$ was used to screen the differences between tumor samples and normal samples in the respective datasets. A $\log _{2}$ (foldchange) $|>1|$ and FDR (False Discovery Rate) $<0.05$ were used as the cut-off criteria for the DEGs. The common DEGs in the GSE94349 and GSE68015 datasets were identified via the intersection function in R. The difference in MMP12 expression between the two groups was compared using the ggplot2 $\mathrm{R}$ package (versions 3.3.3; Bioconductor).

Function and pathway analyses of DEGs. The clusterProfiler software package (versions 3. 18.1; Bioconductor) was used to perform Gene Ontology (GO) and Kyoto Encyclopedia of Genes and Genomes (KEGG) pathway enrichment analyses. GO includes biological processes (BP), cellular components (CC) and molecular functions (MF) (36). Enrichment analysis was performed to determine the biological significance of the DEGs.
Interaction and regulatory network establishment. The Search Tool for the Retrieval of Interacting Genes/ Proteins database (https://www.researchgate.net/figure/ STRING-Search-Tool-for-the-Retrieval-of-Interacting-GenesProteins-network-analysis-of_fig2_234098958) was used to construct the protein-protein interaction (PPI) network of the DEGs, and an interaction score of 0.4 was set as the threshold. The MOCD plug-in version 3.6.1 (Cytoscape) was used to select the aim module: Modules with MCODE scores $>5$, degree cut-offof 2 , node score cut-off of 0.2 , max depth $=100$ and $\mathrm{k}$-score $=2$ were presented. Hub genes were screened out if they had a degree of connectivity $\geq 42$.

Patients and sample collection. Tumor samples were collected and preserved following surgery at the Department of Neurosurgery, The First Affiliated Hospital of Nanchang University (NanChang, China). Tumor samples were collected after nasal endoscopic resection and stored at $4^{\circ} \mathrm{C}$ for transporting. A total of six tumor samples, from three men and three women were obtained. The mean age of the patients was 36 years (age range, 8-68 years), including three men and three women. Patients pathologically diagnosed with ACP were included in the present study, while patients who were associated with other diagnoses were excluded. All specimens were pathologically and clinically diagnosed as ACP by three pathologists. The present study was approved by The Research Ethics Committee of Nanchang University [NanChang, China; First Affiliated Hospital of Nanchang University (2020) Medical Research Ethics Review (No. 160)] and written informed consent was provided by all patients prior to the study start.

Primary cell culture. Fluorescence microscopy (magnification, $\mathrm{x} 200$ ) was performed to confirm the tumor parenchyma for the subsequent steps. Tissue samples were repeatedly washed with PBS and subsequently cut into $2-3 \mathrm{~mm}^{3}$ thick sections. Tissue section were digested using trypsin containing streptomycin and penicillin $(0.25 \%)$ and collagenase II $(1 \mathrm{mg} / \mathrm{ml})$ for $45 \mathrm{~min}$ (all purchased from Beijing Solarbio Science \& Technology Co., Ltd.). The samples were centrifuged at $1,000 \mathrm{x} \mathrm{g}$ for $10 \mathrm{~min}$ at $4^{\circ} \mathrm{C}$, and the supernatant was discarded. Cells were resuspended in high glucose medium (Beijing Solarbio Science \& Technology Co., Ltd.) supplemented with $15 \%$ fetal bovine serum (FBS, Gibco; Thermo Fisher Scientific, Inc.) and incubated at $37^{\circ} \mathrm{C}$ with $5 \% \mathrm{CO}_{2}$.

Immunofluorescence. ACP cells were washed with PBS and fixed in $75 \%$ ethanol for $20 \mathrm{~min}$ at room temperature. Cells were re-washed and blocked with $5 \% \mathrm{BSA}$ at $37^{\circ} \mathrm{C}$ for $1 \mathrm{~h}$. Cells were subsequently incubated with monoclonal anti-pan-cytokeratin (pan-CK, 1:100; cat. no. ab215838; Abcam) and anti-vimentin (VIM; 1:100; cat. no. J144; Invitrogen; Thermo Fisher Scientific, Inc.) antibodies overnight at $4^{\circ} \mathrm{C}$. Following incubation, cells were counterstained with DAPI $(10 \mu \mathrm{g} / \mathrm{ml}$; Sigma-Aldrich; Merck $\mathrm{KGaA}$ ) at $23^{\circ} \mathrm{C}$ for $10 \mathrm{~min}$ and observed under a confocal laser scanning microscope (fluorescence microscope, $\mathrm{x} 200)$. For the MMP408 and control groups, anti- $\beta$-catenin (1:100; cat. no. C7207; Sigma-Aldrich; Merck KGaA) was used.

Cell proliferation assay. ACP cells were seeded into 24-well plates at a density of $3 \times 10^{3}$ cells/well, and in 9 wells, cells 
were treated with $2 \mathrm{nmol} / \mathrm{ml} \mathrm{MMP408}\left(37^{\circ} \mathrm{C}\right.$, Sigma-Aldrich; Merck KGaA) for 24,48 or $72 \mathrm{~h}$ (three wells/condition) as the treatment group, and the remaining wells were treated for different periods of time $(24,48$ or $72 \mathrm{~h})$ at $37^{\circ} \mathrm{C}$, as the control group. DMEM solution containing $10 \mu \mathrm{l}$ Cell Counting Kit-8 (CCK-8, 10:1, cat. no. 40203ES60; Shanghai Yeasen Biotechnology Co., Ltd.) was added to each well and cells were incubated for an additional $2.5 \mathrm{~h}$ in the dark. Cell proliferation was subsequently analyzed at a wavelength of $450 \mathrm{~nm}$, using a microplate reader (Enspire 23001489, PerkinElmer, Inc.). The number of cells in each treatment condition was determined, based on the absorbance.

Cell migration assay. The wound healing assay was performed to assess the migratory ability of ACP cells. ACP cells were seeded into 6 -well plates at a density of $5 \times 10^{5}$ cells/well and incubated in media supplemented with $15 \%$ FBS (37) (Gibco; Thermo Fisher Scientific, Inc.), at $37^{\circ} \mathrm{C}$ for $4 \mathrm{~h}$. Once the cells reached confluence (the cells cover the plate), the monolayers were scratched with a $200 \mu 1$ pipette tip and washed three times with PBS. Cells were incubated in DMEM supplemented with $15 \%$ FBS (37) at $37^{\circ} \mathrm{C}$ for $48 \mathrm{~h}$ (Gibco; Thermo Fisher Scientific, Inc.) and the treatment group was treated with $2 \mathrm{nmol} / \mathrm{ml} \mathrm{MMP408} \mathrm{(Sigma-Aldrich;} \mathrm{Merck} \mathrm{KGaA)} \mathrm{at} 37^{\circ} \mathrm{C}$ for $48 \mathrm{~g}$. Images were taken at 0 and $48 \mathrm{~h}$ using an inverted fluorescence microscope (magnification, x200).

Colony formation assay. ACP cells were seeded into6-wells plates at a density of 500 cells/well, with three wells as the control group and the other three wells as the treatment group. All cells were incubated in DMEM supplemented with $15 \%$ FBS (37) (Gibco; Thermo Fisher Scientific, Inc.). at $37^{\circ} \mathrm{C}$ for $24 \mathrm{~h}$. The treatment groups were treated with $2 \mathrm{nmol} / \mathrm{ml}$ MMP408 at $37^{\circ} \mathrm{C}$ for 2 weeks (Sigma-Aldrich; Merck KGaA). Cells were cultured for 2 weeks, fixed with $4 \%$ paraformaldehyde for $30 \mathrm{~min}$ at $23^{\circ} \mathrm{C}$ stained with $50 \%$ crystal violet dye solution at $23^{\circ} \mathrm{C}$ for $2 \mathrm{~h}$ and imaged. Cell colonies were observed under a fluorescence microscope (magnification, $\mathrm{x} 200$ ); a colony was counted if it contained $\geq 50$ cells.

Western blotting. Cells were collected on ice $\left(4^{\circ} \mathrm{C}\right)$, lysed using lysis buffer (Beyotime Institute of Biotechnology) and subsequently centrifuged at $12,000 \mathrm{x}$ g for $30 \mathrm{~min}$ at $23^{\circ} \mathrm{C}$ to retain the supernatant. The BCA protein analysis kit (Beyotime Institute of Biotechnology) was used to determine protein concentration. Protein lysates $(45 \mu \mathrm{g})$ were separated by SDS-PAGE (7.5\%), transferred onto PVDF (EMD Millipore) membranes and blocked with $5 \%$ skimmed milk at $4{ }^{\circ} \mathrm{C}$ overnight. The membranes were incubated with primary antibodies against c-Myc (1:1,000; cat. no. 10828-1-AP; ProteinTech Group, Inc.), c-Jun (1:2,000; cat. no. 24909-1-AP; ProteinTech Group, Inc.), Wisp1 (1:1,000; cat. no. 18166-1-AP; ProteinTech Group, Inc.), $\beta$-catenin (1:5,000; cat. no. 51067-2-AP; ProteinTech Group, Inc.) and GAPDH (1:5,000; cat. no. 10494-1-AP; ProteinTech Group, Inc.) overnight at $4^{\circ} \mathrm{C}$. The membranes were washed three times with tris-buffered saline with Tween-20 (for $10 \mathrm{~min}$ each) and subsequently incubated with HRP-conjugate goat anti-rabbit IgG secondary antibody (1:10,000; cat. no. 31460; Thermo Fisher Scientific, Inc.). Protein bands were visualized using enhanced chemiluminescence reagent (EMD Millipore) and densitometry analysis was performed using Image J software (Image-Pro Plus 6.0; National Institutes of Health) and the data were normalized to expression of the internal control GAPDH.

Statistical analysis. Statistical analysis was performed using SPSS version 19.0 software (IBM Corp.). All experiments were performed in triplicate and data are presented as the mean \pm standard deviation. Paired Student's t-test was used to compare differences between two groups, while one-way ANOVA followed by Tukey's post hoc test were used to compare differences between multiple groups. $\mathrm{P}<0.05$ was considered to indicate a statistically significant difference.

\section{Results}

Identification of DEGs. The heatmaps and volcano plots were generated to identify the downregulated and upregulated genes in the GEO datasets (GSE94394 and GSE68015). As presented in Fig. 1A, MMP12 expression in the GSE94394 dataset was significantly higher in the normal group compared with the ACP group $(\sim 1,000 x ; P<0.05)$. As presented in Fig. 1B, MMP12 expression in the GSE68015 dataset was significantly higher in the ACP group compared with the normal group $(\sim 1,700 x ; P<0.05)$. The differences in gene expression between the normal and ACP groups are presented in Fig. 1C and D.

MMP12 expression in the ACP group compared with the normal group and construction of the PPI network. MMP12 expression levels in the two datasets are presented in Fig. 2E and F. The results demonstrated that MMP12 was significantly higher in the ACP group compared with the normal group $(\mathrm{P}<0.05)$, which is consistent with a previous study (9). The PPI networks are presented in Fig. 2G and H. Although MMP12 was not one of the top 10 dysregulated genes (top 26 and 42 in the GSE94394 and GSE68015 datasets, respectively), the results demonstrated that MMP12 has complex associations with several other genes.

GO and KEGG pathway enrichment analyses of the DEGs. GO and KEGG pathway enrichment analyses were performed using the clusterProfiler package, with a threshold of $\mathrm{P}<0.05$ (Fig. 2A). Enriched BP, CC and MF terms were used to obtain a better understanding of the biological functions of overlapping DEGs. The results demonstrated that the significantly enriched GO terms for BP were 'neutrophil activation', 'neutrophil mediated immunity', 'neutrophil activation involved in immune response', 'neutrophil degranulation', 'regulation of neuron projection development', 'regulation of trans-synaptic signaling', 'modulation of chemical synaptic transmission', 'axonogenesis', 'synapse organization' and 'regulation of intracellular transport' (Fig. 2B). Furthermore, the significantly enriched $\mathrm{GO}$ terms for $\mathrm{CC}$ were 'neuronal cell body', 'presynapse', 'cell-substrate junction', 'cell-substrate adherens junction', 'focal adhesion', 'postsynaptic specialization', 'neuron to neuron synapse', 'glutamatergic synapse', 'asymmetric synapse' and 'postsynaptic density' (Fig. 2B). The significantly enriched GO terms for MF were 'cell adhesion molecule binding', 'small GTPase binding', 'Ras GTPase binding', 'actin binding', 'cadherin binding', 'tubulin 

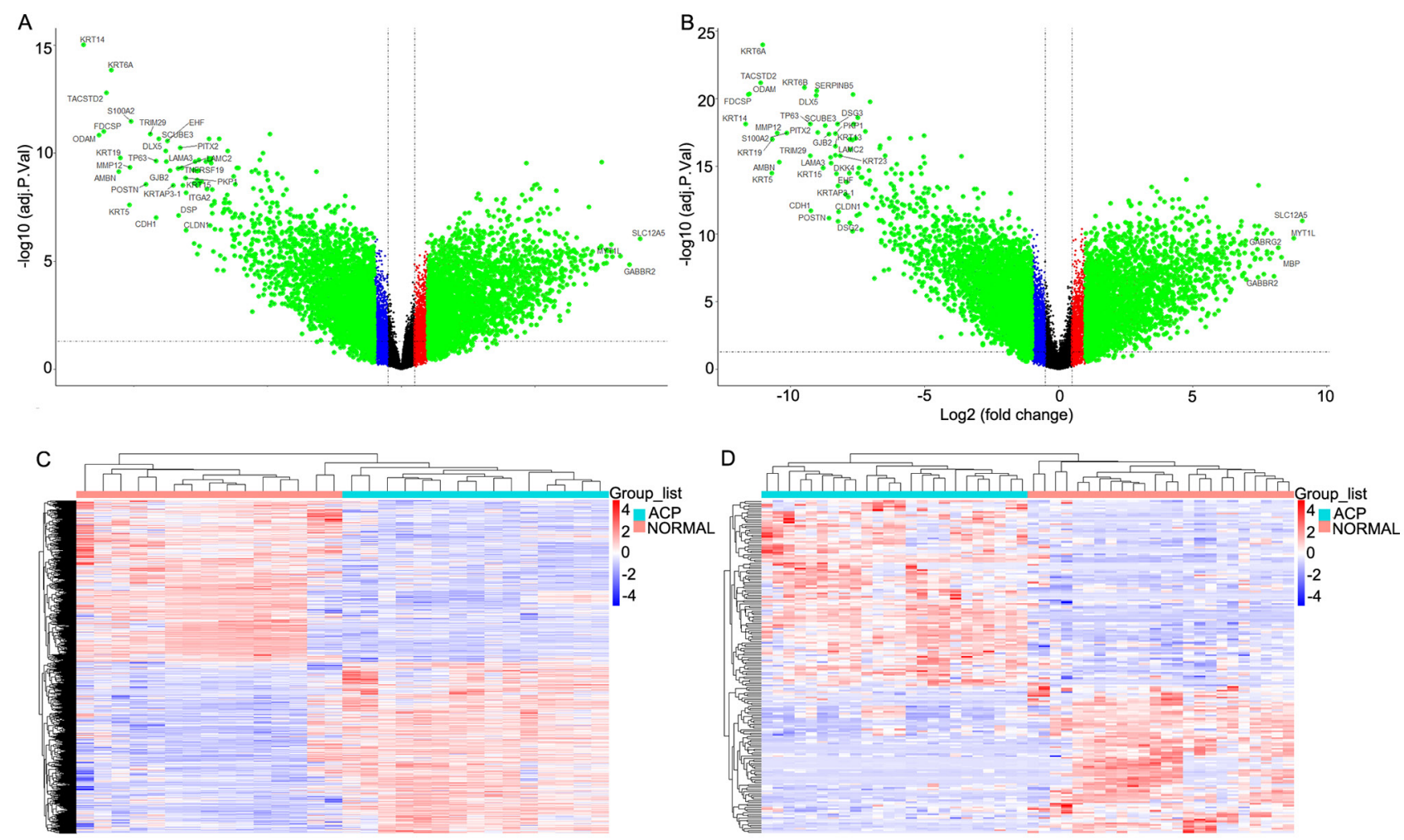

Figure 1. Hierarchical cluster analysis (heatmaps) and volcano plots of the common DEGs between ACP tissues and normal tissues. Hierarchical cluster analysis in the (A and C) GSE68015 and (B and D) GSE94349 datasets. DEGs were defined as P<0.05 and $\log _{2}$ (fold change) $>11.0$. DEGs, differentially expressed genes; ACP, adamantinomatous craniopharyngioma.

binding', 'protein C-terminus binding', 'Rho GTPase binding', 'extracellular matrix structural constituent' and 'SNARE binding' (Fig. 2B). KEGG pathway enrichment analysis demonstrated that the DEGs were primarily enriched in the 'MAPK signaling pathway', 'chemokine signaling pathway', 'endocrine resistance', 'ErbB signaling pathway', 'EGFR tyrosine kinase inhibitor resistance', 'N-glycan biosynthesis', 'human papillomavirus infection', 'proteoglycans in cancer', 'human T-cell leukemia virus 1 infection', 'salmonella infection', 'focal adhesion', 'axon guidance', 'hippo signaling pathway', 'cellular senescence', 'glutamatergic synapse' and 'AGE-RAGE signaling pathway' in diabetic complications (Fig. 2C and D). These significantly enriched terms and pathways may provide further insight into potentially druggable targets for management of DEGs.

Establishment and identification of an ACP cell line. Primary ACP cells which were obtained from tissues pathologically confirmed to be ACP were successfully cultured for subsequent functional assays in vitro. Keratins are established molecular markers for tumors of an epithelial origin (38-40) and Vim is an important marker to identify epithelial tumor cells because epithelial tumors do not express Vim (41). Thus, pan-CK and VIM expression levels were used as positive and negative controls of analysis of the primary ACP cell via immunofluorescence analysis. The results demonstrated that pan-CK was primarily expressed in the cytoplasm of ACP cells (Fig. 3A; left), while VIM expression was not observed (Fig. 3A; right). These results suggest that the primary cells were ACP cells, and they were not contaminated by $\mathrm{CP}$ associated fibroblasts.
MMP12 promotes ACP proliferation and migration in vitro. To investigate the effect of MMP408 on the proliferation and migration of ACP cells, the CCK-8, colony formation and wound healing assays were performed. The results of the CCK-8 assay demonstrated that MMP408 significantly decreased the proliferation of ACP cells compared with the control group $(\mathrm{P}<0.05$; Fig. $3 \mathrm{~B})$. The results of the colony formation assay indicated that the number of cell colonies in the treated group was significantly lower compared with the control group $(\mathrm{P}<0.001$; Fig. $3 \mathrm{C})$. The results of the wound healing assay demonstrated that MMP408 significantly inhibited the migratory ability of ACP cells ( $\mathrm{P}<0.001$; Fig. 4A).

MMP12 promotes the expression levels of $\beta$-catenin, $c-M y c$ and Wispl. The Wnt/ $\beta$-catenin signaling pathway is involved in the progression and development of several types of tumors (42). $\beta$-catenin serves an important role in tumor invasion and development. MMP12 may interact with the Wnt/ $\beta$-catenin signaling pathway to regulate the development of the central nervous system (43). c-Myc expression was also assessed in the present study. The results demonstrated that MMP408 significantly decreased the protein expression levels of c-Myc, Wisp1 and $\beta$-catenin $(\mathrm{P}<0.01$ and $\mathrm{P}<0.001)$, but not c-Jun (Fig. 4B).

Immunofluorescence analysis indicated that treatment with MMP408 decreased $\beta$-catenin expression in the nucleus (Fig. 4C), where it normally interacts with target genes involved in tumor progression (30-32). Taken together, these results suggest that MMP12 may promote the translocation of $\beta$-catenin into the nucleus by regulating $\beta$-catenin and c-Myc, 


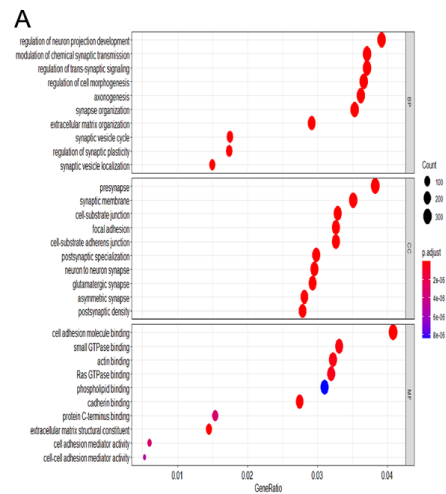

C

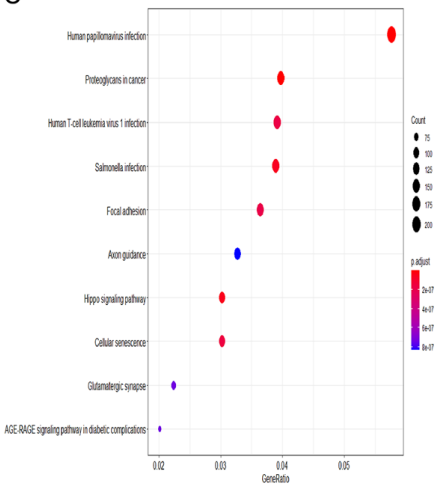

B

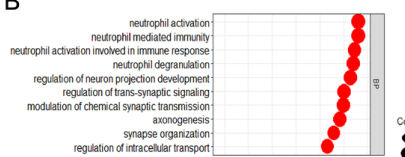

E
F

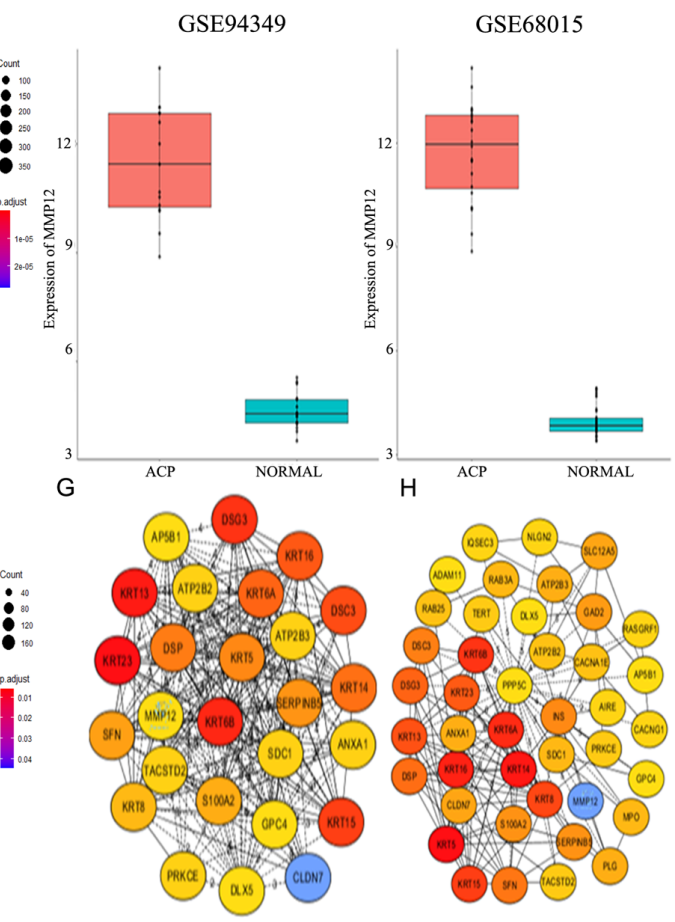

Figure 2. Gene Ontology and Kyoto Encyclopedia of Genes and Genomes pathway enrichment analyses were performed using the clusterProfiler package with a threshold of $\mathrm{P}<0.05$ for differentially expressed genes between ACP tissues and normal tissues. Analysis of the (A and C) GSE68015 and (B and D) GSE94349 datasets. (E and F) MMP12 expression in ACP tissues and normal tissues. (G and H) Protein-protein interaction networks of MMP12. Modules with MCODE scores $>5$, degree cut-off of 2, node score cut-off of 0.2 , max depth $=100$ and k-score of 2 are presented. Hub genes were screened if they exhibited a degree of connectivity $\geq 42$. ACP, adamantinomatous craniopharyngioma; MMP, matrix metalloproteinase.

A
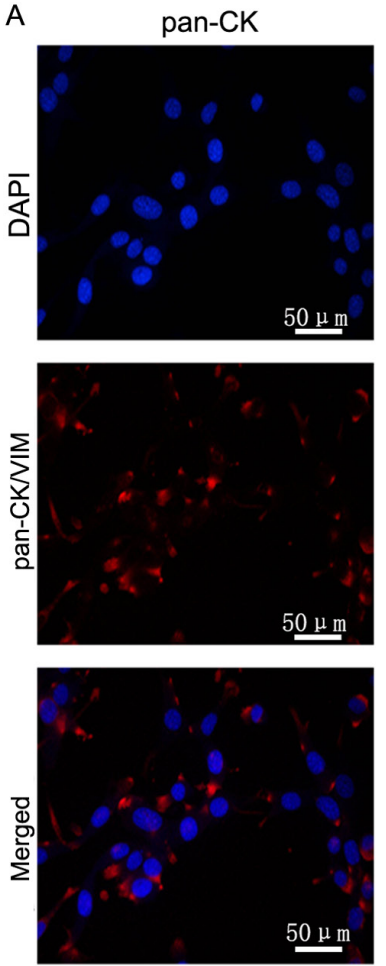

VIM
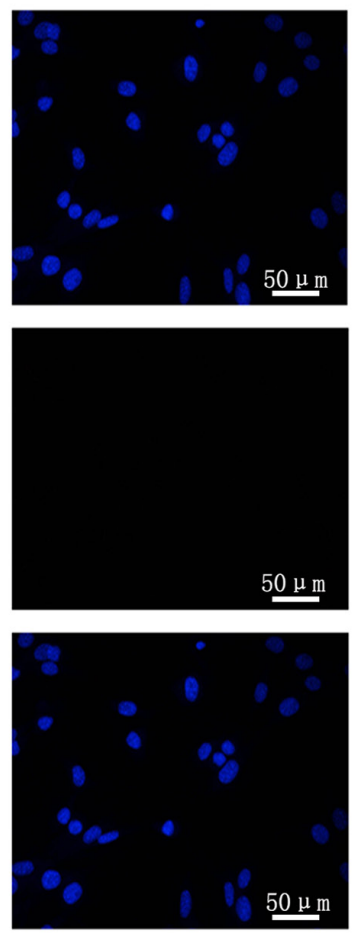

B
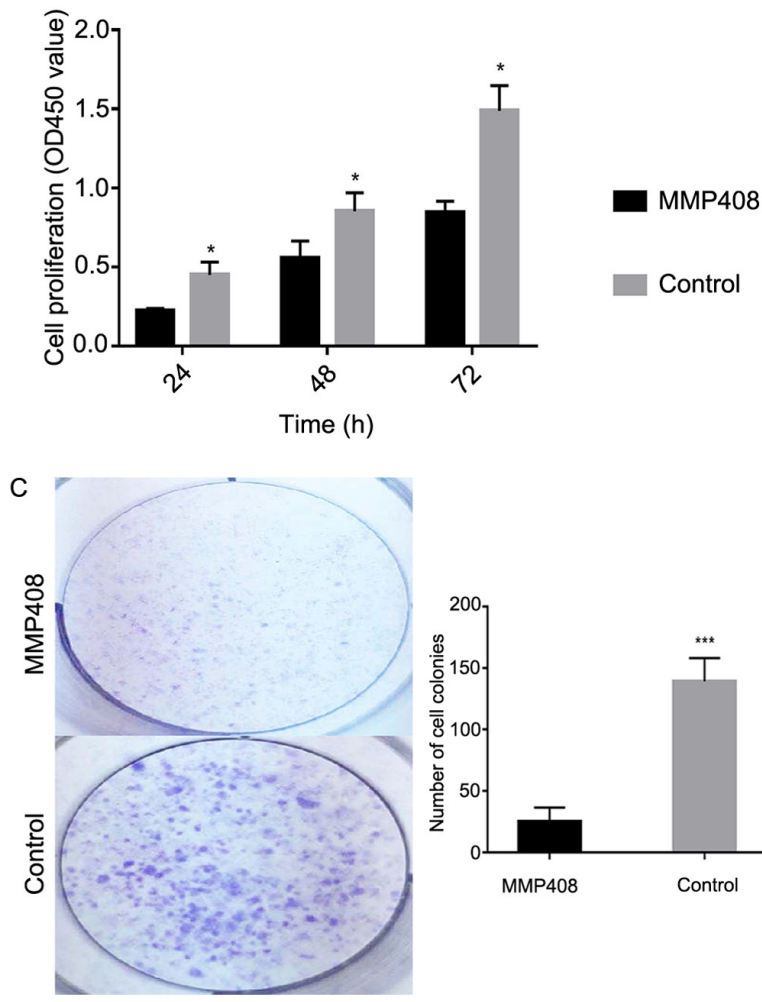

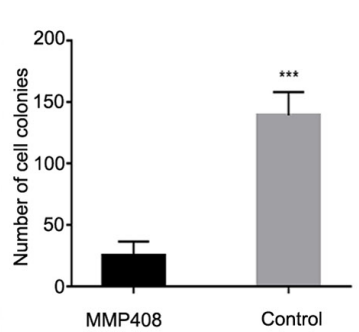

Figure 3. Immunofluorescence, Cell Counting-Kit 8 and colony formation analyses of ACP. (A) Immunofluorescence analysis of primary ACP cells. Pan-CK and VIM expression levels were determined in cells counterstained with DAPI. (B) The Cell Counting-Kit 8 assay was performed to assess the effect of MMP408 on ACP cell proliferation. (C) MMP12 inhibited colony formation of ACP cells. ${ }^{*} \mathrm{P}<0.05 ;{ }^{* * *} \mathrm{P}<0.001$ vs. MMP408. ACP, adamantinomatous craniopharyngiomas; pan-CK, pan-cytokeratin; VIM, vimentin; MMP, matrix metalloproteinase; OD, optical density. 


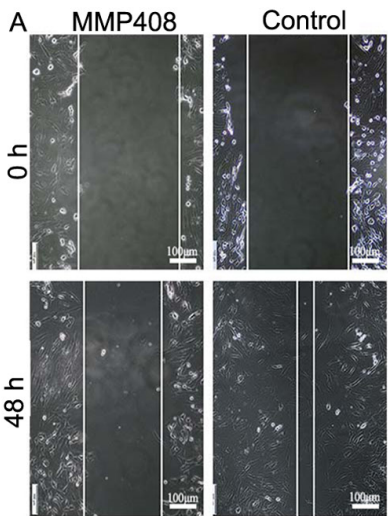

B

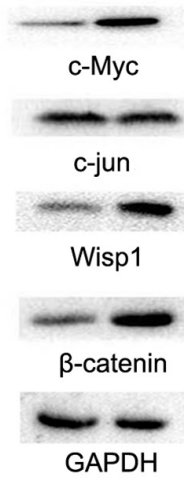

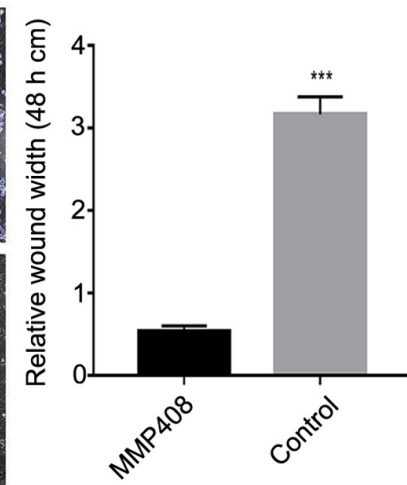

C
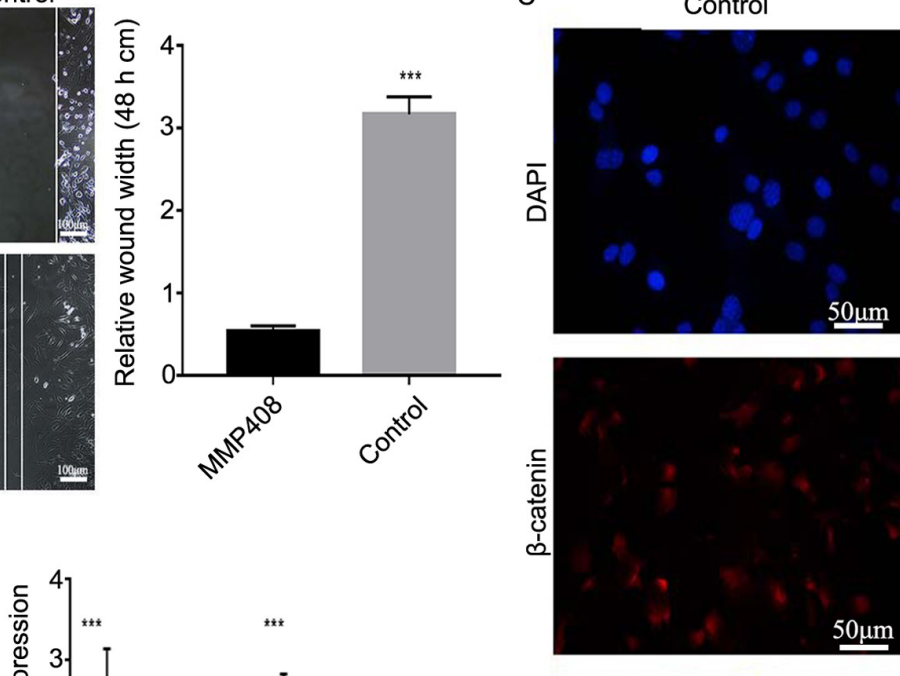

MMP408

Control

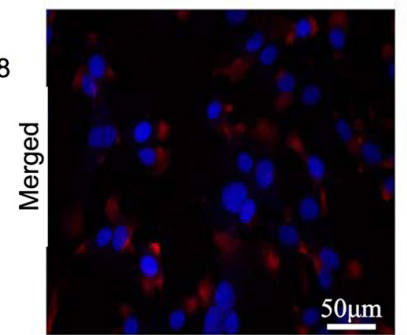

MMP408
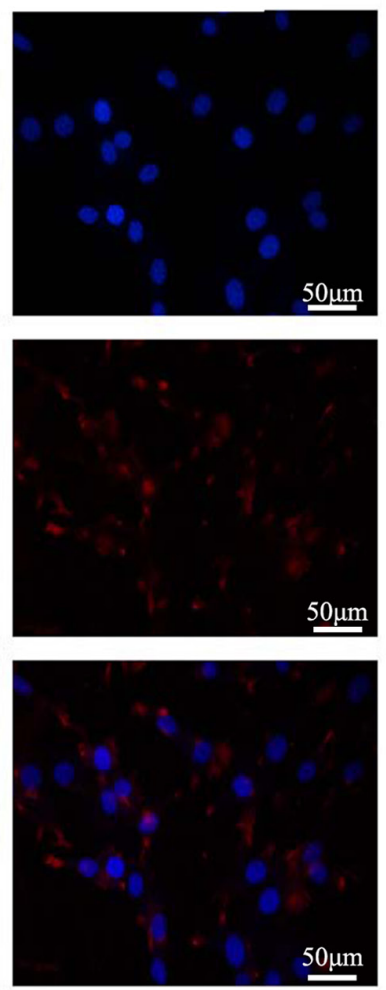

Figure 4. Wound healing assay and western blot and immunofluorescence analyses of ACP. (A) The wound healing assay was performed to assess the effect of MMP408 on the migratory ability of primary ACP cells. (B) Western blot analysis was performed to assess the effect of MMP408 on the protein expression levels of $\beta$-catenin, c-Myc, c-Jun and Wisp1. (C) Immunofluorescence analysis was performed to assess the effect of MMP408 on the distribution of $\beta$-catenin. ${ }^{* *} \mathrm{P}<0.01 ;{ }^{* * *} \mathrm{P}<0.001$ vs. MMP408. MMP, matrix metalloproteinase; ACP, adamantinomatous craniopharyngiomas.

where it drives transcription of its target genes, and induces proliferation and migration of ACP cells.

\section{Discussion}

Although ACP is a benign tumor, it accounts for $3 \%$ of all and $4 \%$ of childhood cranial tumors, with an annual incidence ranging from 5-20 cases per 100,000,000 (44-46). However, due to its unique anatomical structure and the risk of serious postoperative complications $(7,8)$, it is urgent to improve surgical removal and non-surgical treatment methods. However, the number of studies on drugs targeting genes uniquely upregulated in ACP are limited. It has been demonstrated that MAPK/ERK inhibitors can inhibit progression of ACP tumors; however, related research is still in its infancy (45). Thus, further studies are required to understand the unique genome of ACP. In addition, due to various factors, bioinformatics-based analyses of ACP is limited.

In the present study, the gene expression profiles of the GSE94349 and GSE68015 datasets were integrated to identify the DEGs between ACP tissues and normal tissues using bioinformatics analysis. GO and KEGG pathway enrichment analyses were performed using the clusterProfiler $\mathrm{R}$ package. The enriched GO terms for BP were 'neutrophil activation', 'neutrophil mediated immunity' and 'neutrophil activation involved in immune response', and KEGG pathway analysis demonstrated that these DEGs were enriched in the 'chemokine signaling pathway', 'endocrine resistance' and 'human
T-cell leukemia virus 1 infection' in both groups, which are all associated with inflammation in the tumor (32). MMP12 can affect tumor inflammatory response by affecting the secretion and expression of macrophages (46). A previous study reported that inflammation plays a key role in the occurrence and progression of ACP (32). In addition, MMP12 plays an important role in the occurrence and progression of ACP (32). Notably, in the KEGG pathway enrichment analysis, it was confirmed that EGFR tyrosine kinase inhibitor resistance exists in ACP. However, the specific mechanism and situation under which this resistance is exhibited requires further investigation, which may also be relevant for further research and development of targeted drugs for ACP.

The progression of cancer cells primarily manifests as increased cell proliferation and migration $(19,29,37,43,47)$. In the present study, the wound healing, cell proliferation and colony formation assays demonstrated that MMP408 inhibited the proliferative and migratory abilities of ACP cells. Previous studies have demonstrated that MMP408 can inhibit MMP12 expression at both mRNA and protein levels (26-29). Thus, it was speculated that MMP12 can inhibit the proliferation and migration of ACP cells. However, the lack of experimental verification of the inhibition of MMP12 expression/enzymatic activity by MMP408 is a limitation of the present study.

Currently, the predominant hypothesis by which ACP develops/progresses is overactivation of the $\mathrm{Wnt} / \beta$-Catenin signaling pathway; thus, the expression of $\beta$-catenin is 
considered an important indicator of ACP (48). $\beta$-catenin is a cytoplasmic protein that mediates gene transcription and cell-cell adhesion (48). In the absence of the Wnt ligand, $\beta$-catenin undergoes proteasomal degradation as a result of interactions between Axin, glycogen synthase kinase $3 \beta$ and anaphase-promoting complex. $\beta$-catenin is considered an important regulator of ACP, and in most tumors, c-Myc promotes the proliferation of cancer cells by acting on SLC1A5. Jun can regulate tumor proliferation and other functions via AP-1. It has been reported that high Wisp1 expression is positively associated with the proliferation and invasion of several types of tumors, and is negatively associated with prognosis. $\beta$-catenin is prevented from activating the transcription factors T-cell factor (TCF) and lymphoid enhancer factor (LEF), and c-Myc, c-Jun and Wisp1 are downstream proteins of TCF/LEF $(32,47-49)$. The expression of these genes has important effects on ACP cell functions, such as proliferation and invasion $(32,47-49)$. In the present study, immunofluorescence and western blot analyses demonstrated that the expression levels of $\beta$-catenin, c-Myc and Wisp1 significantly decreased in ACP cells when MMP12 expression was inhibited. Thus, inhibition of MMP12 can inhibit the transcription and expression of related proteins in $\mathrm{ACP}$, and thus inhibit the occurrence and progression of ACP.

Recent studies have reported that MMP12 expression in ACP is $>820 x$ of that in the surrounding normal tissues, and thus may be an important potential target for ACP therapy in the future $(9,38,48)$. In the present study, MMP12 expression significantly varied between the ACP and normal groups. Although MMP12 was not amongst the top 10 genes that were differentially expressed between the ACP and normal groups, it did not affect the role of MMP12 in ACP, as there were several associations between MMP12 and the top 10 dysregulated genes.

MMP12 is predominantly expressed in the cell nuclei and the extracellular matrix $(28,29)$. Previous studies $(28,29)$ have reported that MMP12 possesses dual functions. Its expression in the cell nucleus can promote tumor proliferation, while its expression in the extracellular matrix can inhibit tumor growth (9). Thus, determining the mode of action of MMP12 in ACP is important in any study of its role in cancer. In the present study, inhibiting MMP12 expression decreased the proliferative and migratory abilities of ACP cells. In addition, the expression levels of the related proteins in vitro decreased, suggesting that MMP12 promotes ACP proliferation and migration in vitro. The effects of MMP12 inhibition should be determined in vivo $(9,50)$.

In conclusion, the present study performed bioinformatics analysis of ACP datasets to determine the important characteristics of ACP. Taken together, these results support the notion that MMP12 serves an important role in ACP and may be involved in drug resistance. The in vitro experiments demonstrated that MMP408 decreased the proliferation and migration of ACP cells, suggesting the therapeutic potential of targeting MMP12 in management of ACP. However, further studies are required to validate the results presented here.

\section{Acknowledgements}

Not applicable.

\section{Funding}

The present study was funded by National Natural Science Foundation of China (grant no. 82060246).

\section{Availability of data and materials}

The datasets used and/or analyzed during the present study are available from the corresponding author upon reasonable request.

\section{Authors' contributions}

ML and TH conceived and designed the experiments, analyzed the data and prepared the manuscript. ML, LZ and SL performed the experiments. LF, LY, XW, CY, YB, SL, ZT, SZ, BT, EZ, SX and CC contributed to data collection and analysis. TH and ML and LZ confirmed the authenticity of all the raw data. All authors have read and approved the final version.

\section{Ethics approval and consent to participate}

The present study was approved by The Research Ethics Committee of Nanchang University [First Affiliated Hospital of Nanchang University (2020) Medical Research Ethics Review (No. 160)] and written informed consent was provided by all patients prior to the study start.

\section{Patient consent for publication}

Not applicable.

\section{Competing interests}

The authors declare that they have no competing interests.

\section{References}

1. Martinez-Barbera JP and Buslei R: Adamantinomatous craniopharyngioma: Pathology, molecular genetics and mouse models. J Pediatr Endocrinol Metab 28: 7-17, 2015.

2. Kasai H, Hirano A,Llena JF and Kawamoto K: A histopathological study of craniopharyngioma with special reference to its stroma and surrounding tissue. Brain Tumor Pathol 14: 41-45, 1997.

3. Barkhoudarian $G$ and Laws ER: Craniopharyngioma: History. Pituitary 16: 1-8, 2013.

4. Laws ER Jr: Transsphenoidal microsurgery in the management of craniopharyngioma. J Neurosurg 52: 661-666, 1980.

5. Laws ER Jr: Transsphenoidal removal of craniopharyngioma. Pediatr Neurosurg 21 (Suppl 1): S57-S63, 1994.

6. Yaşargil MG, Curcic M, Kis M, Siegenthaler G, Teddy PJ and Roth P: Total removal of craniopharyngiomas. Approaches and long-term results in 144 patients. J Neurosurg 73: 3-11, 1990.

7. Alvarez M: Craniopharyngiomas. J Neurosci Nurs 38: 362-368, 2006.

8. Karavitaki N, Cudlip S, Adams CB and Wass JA: Craniopharyngiomas. Endocr Rev 27: 371-397, 2006.

9. Gump JM, Donson AM, Birks DK, Amani VM, Rao KK, Griesinger AM, Kleinschmidt-DeMasters BK, Johnston JM, Anderson RC, Rosenfeld A, et al: Identification of targets for rational pharmacological therapy in childhood craniopharyngioma Acta Neuropathol Commun 3: 30, 2015.

10. Xia Z, Liu W, Li S, Jia G, Zhang Y, Li C, Ma Z, Tian J and Gong J: Expression of matrix metalloproteinase-9, type IV collagen and vascular endothelial growth factor in adamantinous craniopharyngioma. Neurochem Res 36: 2346-2351, 2011. 
11. Baker JC, Beddington RS and Harland RM: Wnt signaling in Xenopus embryos inhibits bmp4 expression and activates neural development. Genes Dev 13: 3149-3159, 1999.

12. Li T, Gao X, Han L, Yu J and Li H: Identification of hub genes with prognostic values in gastric cancer by bioinformatics analysis. World J Surg Oncol 16: 114, 2018.

13. Wang K, Yuen ST, Xu J, Lee SP, Yan HH, Shi ST, Siu HC, Deng S, Chu KM, Law S, et al: Whole-genome sequencing and comprehensive molecular profiling identify new driver mutations in gastric cancer. Nat Genet 46: 573-582, 2014.

14. Jiang P and Liu XS: Big data mining yields novel insights on cancer. Nat Genet 47: 103-104, 2015.

15. Tang F, Qian X, Lu Z, Lai Y, Li Z, He C and He Z: Identification of differentially expressed genes, biological pathways and prognostic signature in bladder cancer. BMC Urology: 10.21203/rs.3.rs-362542/v1.

16. Werb Z and Gordon S: Elastase secretion by stimulated macrophages. Characterization and regulation. J Exp Med 42: 361-377, 1975.

17. Banda MJ and Werb Z: Mouse macrophage elastase. Purifcation and characterization as a metalloproteinase. Biochem J 193: $589-605,1981$.

18. White RR, Norby D, Janoff A and Dearing R: Partial purifcation and characterization of mouse peritoneal exudative macrophage elastase. Biochim Biophys Acta 612: 233-244, 1980.

19. Hautamaki RD, Kobayashi DK, Senior RM and Shapiro SD Requirement for macrophage elastase for cigarette smoke-induced emphysema in mice. Science 277: 2002-2004, 1997.

20. Shipley JM, Wesselschmidt RL, Kobayashi DK, Ley TJ and Shapiro SD: Metalloelastase is required for macrophagemediated proteolysis and matrix invasion in mice. Proc Natl Acad Sci USA 93: 3942-3946, 1996.

21. Marchant DJ, Bellac CL, Moraes TJ, Wadsworth SJ, Dufour A, Butler GS, Bilawchuk LM, Hendry RG, Robertson AG, Cheung CT, et al: A new transcriptional role for matrix metalloproteinase-12 in antiviral immunity. Nat Med 20: 493-502, 2014.

22. Kerkelä E, Ala-Aho R, Jeskanen L, Rechardt O, Grénman R, Shapiro SD, Kähäri VM and Saarialho-Kere U: Expression of human macrophage metalloelastase (MMP-12) by tumor cells in skin cancer. J Invest Dermatol 114: 1113-1119, 2000.

23. Zhao X, Xu M, Cai Z, Yuan W, Cui W and Li MD: Identifcation of LIFR, PIK3R1, and MMP12 as novel prognostic signatures in gallbladder cancer using network-based module analysis. Front Oncol 9: 325, 2019.

24. Roman J: On the 'TRAIL' of a killer: MMP12 in lung cancer. Am J Respir Crit Care Med 196: 262-264, 2017.

25. Klupp F, Neumann L, Kahlert C, Diers J, Halama N, Franz C, Schmidt T, Koch M, Weitz J, Schneider M and Ulrich A: Serum MMP7, MMP10 and MMP12 level as negative prognostic markers in colon cancer patients. BMC Cancer 16: 494 2016.

26. Ng KT, Qi X, Kong KL, Cheung BY, Lo CM, Poon RT, Fan ST and Man K: Overexpression of matrix metalloproteinase-12 (MMP-12) correlates with poor prognosis of hepatocellular carcinoma. Eur J Cancer 47: 2299-2305, 2011.

27. Ella E, Harel Y, Abraham M, Wald H, Benny O, Karsch-Bluman A, Vincent D, Laurent D, Amir G, Izhar U, et al: Matrix metalloproteinase 12 promotes tumor propagation in the lung. J Thorac Cardiovasc Surg 155: 2164-2175, 2018.

28. Yang W, Arii S, Gorrin-Rivas MJ, Mori A, Onodera H and Imamura M: Human macrophage metalloelastase gene expression in colorectal carcinoma and its clinicopathologic signifcance. Cancer 91: 1277-1283, 2001.

29. Kerkelä E, Ala-aho R, Klemi P, Grénman S, Shapiro SD Kähäri VM and Saarialho-Kere U: Metalloelastase (MMP-12) expression by tumour cells in squamous cell carcinoma of the vulva correlates with invasiveness, while that by macrophages predicts better outcome. J Pathol 198: 258-269, 2002.

30. Chen S, Xie J, Zhao K, Ren L, Deng Y, Xie X, Chen S, Xu H Long $X$ and Liu E: LPS aggravates lung inflammation induced by RSV by promoting the ERK-MMP-12 signaling pathway in mice. Respir Res 21: 193, 2020.
31. Li J, Wang JJ, Peng Q, Chen C, Humphrey MB, Heinecke J and Zhang SX: Macrophage metalloelastase (MMP-12) deficiency mitigates retinal inflammation and pathological angiogenesis in ischemic retinopathy. PLoS One 7: e52699, 2012.

32. Apps JR, Carreno G, Gonzalez-Meljem JM, Haston S, Guiho R, Cooper JE, Manshaei S, Jani N, Hölsken A, Pettorini B, et al: Tumour compartment transcriptomics demonstrates the activation of inflammatory and odontogenic programmes in human adamantinomatous craniopharyngioma and identifies the MAPK/ERK pathway as a novel therapeutic target. Acta Neuropathol 135: 757-777, 2018.

33. Gupta S, Bi WL, Giantini Larsen A, Al-Abdulmohsen S, Abedalthagafi $\mathrm{M}$ and Dunn IF: Craniopharyngioma: A roadmap for scientific translation. Neurosurg Focus 44: E12, 2018.

34. Gene Expression Omnibus: Series GSE94349. https://www. ncbi.nlm.nih.gov/geo/query/acc.cgi?acc=GSE94349. Accessed February 1, 2017.

35. Gene Expression Omnibus: Series GSE68015. https://www.ncbi. nlm.nih.gov/geo/query/acc.cgi?acc=GSE68015. Accessed April $18,2015$.

36. Wang F, Xue Q, Xu D, Jiang Y, Tang C and Liu X: Identifying the hub gene in gastric cancer by bioinformatics analysis and in vitro experiments. Cell Cycle 19: 1326-1337, 2020.

37. Yin X, Liu Z, Zhu P, Wang Y, Ren Q, Chen $\mathrm{H}$ and $\mathrm{Xu} \mathrm{J}$ : CXCL12/CXCR4 promotes proliferation, migration, and invasion of adamantinomatous craniopharyngiomas via PI3K/AKT signal pathway. J Cell Biochem 120: 9724-9736, 2018.

38. Adamson TE, Wiestler OD, Kleihues P and Yasargil MG: Correlation of clinical and pathological features in surgically treated craniopharyngiomas. J Neurosurg 73: 12-17, 1990.

39. Nelson WG, Battifora H, Santana H and Sun TT: Specific keratins as molecular markers for neoplasms with a stratified epithelial origin. Cancer Res 44: 1600-1603, 1984.

40. Quentmeier H, Osborn M, Reinhardt J, Zaborski M and Drexler HG: Immunocytochemical analysis of cell lines derived from solid tumors. J Histochem Cytochem 49: 1369-1378, 2001.

41. Zhang N: Vimentin and tumor diagnosis. Zhonghua Bing Li Xue Za Zhi 19: 122-124, 1990 (In Chinese).

42. Zimmerli D, Hausmann G, Cantù $\mathrm{C}$ and Basler $\mathrm{K}$ : Pharmacological interventions in the Wnt pathway: Inhibition of Wnt secretion versus disrupting the protein-protein interfaces of nuclear factors. Br J Pharmacol 174: 4600-4610, 2017.

43. Chelluboina B, Nalamolu KR, Klopfenstein JD, Pinson DM, Wang DZ, Vemuganti R and Veeravalli KK: MMP-12, a promising therapeutic target for neurological diseases. Mol Neurobiol 55: 1405-1409, 2017.

44. Bunin GR, Surawicz TS, Witman PA, Preston-Martin S, Davis F and Bruner JM: The descriptive epidemiology of craniopharyngioma. Neurosurg Focus 3: e1, 1997.

45. Erfurth EM, Holmer H and Fjalldal SB: Mortality and morbidity in adult craniopharyngioma. Pituitary 16: 46-55, 2013.

46. Ostrom QT, Gittleman H, Farah P, Ondracek A, Chen Y, Wolinsky Y, Stroup NE, Kruchko C and Barnholtz-Sloan JS: CBTRUS statistical report: Primary brain and central nervous system tumors diagnosed in the United States in 2006-2010. Neuro Oncol 15 (Suppl 2): ii1-ii56, 2013.

47. Brennan A, Leech JT, Kad NM and Mason JM: Selective antagonism of cJun for cancer therapy. J Exp Clin Cancer Res 39: 184, 2020.

48. Gurbuz I and Chiquet-Ehrismann R: CCN4/WISP1 (WNT1 inducible signaling pathway protein 1): A focus on its role in cancer. Int J Biochem Cell Biol 62: 142-146, 2015.

49. Panda S, Banerjee N and Chatterjee S: Solute carrier proteins and c-Myc: A strong connection in cancer progression. Drug Discovery Today 25: 891-900, 2020.

50. Yang M, Zhang X, Liu Q, Niu T, Jiang L, Li H, Kuang J, Qi C, Zhang Q, He X, et al: Knocking out matrix metalloproteinase 12 causes the accumulation of M2 macrophages in intestinal tumor microenvironment of mice. Cancer Immunol Immunother 69: 1409-1421, 2020.

This work is licensed under a Creative Commons Attribution-NonCommercial-NoDerivatives 4.0 International (CC BY-NC-ND 4.0) License. 